\title{
A Hybrid Method for Weapon System-of-systems Capability Requirement Planning
}

\author{
Ning Gu and Duoyong Sun \\ School of Information System and Management, National University of Defense \\ Technology, Changsha, Hunan Province, China \\ guningguning@hotmail.com
}

\begin{abstract}
Capability-Based requirements management and planning thoughts and methods have gone increasingly deep into the development of Weapon System-of-Systems (WSoS). In the traditional top-to-bottom capability requirement planning (CRP) methods, the constraints among different capabilities indexes acquired from the tasks decomposition are neglected, which leads to the irrationalities and conflicts between different WSoS capability requirement indexes. Based on the analysis and description of capability requirements planning problem, the constraints are divided into five types and extracted from WSoS according multiple views thoughts. In addition, Interval Weighted Constraint Network (IWCN) is introduced and a consistency detection algorithm and a conflicts elimination strategy are proposed to achieve a capabilities requirement plan satisfying the tasks requirements and the capabilities indexes constraints. Finally, the practical applicability within a Joint Operations System of Systems (JOSOS) based on the space force capability requirement scheme planning is demonstrated, which proves the validation of the method built in this study.
\end{abstract}

Keywords: Weapons system-of-systems (WSOS); Capability Requirement Planning $(C R P)$; Interval weighted constraint network (IWCN); Conflicts Elimination Strategy

\section{Introduction}

Weapon system-of-systems(WSoS), composed of various Weapon equipment systems which are linked and influenced by each other, is a higher level system under strategic guidance, operational command and protection conditions for the completion of certain operational tasks [1-2]. As an important measure of WSoS, WSoS capability is the ability to complete a set of tasks with the given mission and achieve the intended effect under the given conditions [3]. WSoS capability is the basis for determining the performance of weapons systems, and WSoS capability requirements analysis plays an important part in the allocation of system resources, requirements definition, structural design, as well as system-of-systems components trade-offs work [2].

As an essential phase of capability requirements management, the capability requirement planning is a systematic project under the guidance of the national defense strategy, to ascertain the expected level of weapon system-of-systems capability in a certain period of time satisfying the strategic mission requirements and system constraints [4]. Obviously, The WSoS will have a total mission goal for the entire macroscopic SoS, however, it is general rather than enough in detail for the individual weapon system to execute. Therefore, in the methods for capability requirements planning and analysis, the mission task decomposition is very common to obtain capability requirements plans [2]. This top-down planning method achieves the tracking and positioning of "requirement flow", but it neglects the conflicts and contradictions among the weapon system-ofsystems capability requirements indexes. The interdependence and the constraints among the various weapon equipment systems and the operational activities which are linked to 
each other, lead to a "Back-tracking" failure between the WSoS capability requirements and the total mission requirements. In other words, the WSoS capability requirements acquired by the decomposition of the operational activities cannot successfully meet all the requirements of the systems.

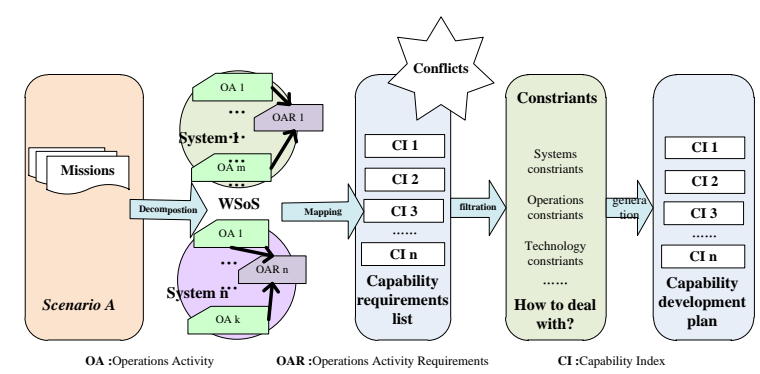

Figure 1. WSoS Capability Requirements Planning Problem Diagram

Thus, the essence of solving the conflicts and contradictions in the list of capability requirements is the optimization of capability requirements from the mission tasks decomposition, to meet a variety of different types of constraints. As shown in Figure 1, the capability requirements plans eventually adapted to the filtration of the constraints will undoubtedly meet a variety of requirements within the individual systems.

The rest of the paper is structured as follows: We first give a brief background required for an understanding of the concerning concept and methodology referred in the following sections. This analysis of WSoS CRP problem includes a short overview of military SoS and its application, a relationship between emergence behavior and WSoS capability, description of WSoS CRP problem in the SoS evolutionary developing process. Next, we propose a definition of conflicts and constraints network based on interval numbers, including $\mathrm{d}$ other main functional models including the definition of IWCN model, Constraint propagation Algorithm and Conflicts elimination strategy.

Subsequently, we present an illustration about Joint Operations System of Systems (JOSoS) based on the space force, we built the IWCN model of the JOSoS as shown in the following to solve its CRP problem. The case proved that the method is available for CRP problem of WSoS. The final plan does not only meet the needs of WSoS construction but also accord with the relationship constraints between equipment as well as that between indexes. Finally, we give a conclusion of this article and future work proposals.

\section{Analysis of WSoS CRP Problem}

\subsection{System of Systems and its Typical Characteristics in Military Application}

Maier lists five hallmarks of SoS[6] which have been taken as common attributes to examine a enterprise belonging to SoS or not. The hallmarks are:

- Operational Independence of Elements

- Managerial Independence of Elements

- Evolutionary Development

- Emergent Behaviour

- Geographical Distribution of Elements

According to the above attributes, military service is a typical SoS which existed several years ago. Many SoS is under developing [5], such as FCS (Future Combat Systems), SIAP (Single Integrated Air Picture), BMDS (Ballistic Missile Defense Systems), et al. De Wolf and Holvoet introduce the noun emergent for the result of a process - in contrast to the process itself - which leads to a certain macroscopic pattern: "A system exhibits emergence when there are coherent emergences at the macro-level that dynamically arise 
from the interactions between the parts at the micro-level. Such emergences are novel with respect to the individual parts of the system." Properties, behavior, structure, or patterns, for instance, can be emergent. "The whole is bigger than the sum of its parts" is taken as the traditional descriptions explaining the notion of emergence. But it is not adequate to describe the underlying meaning of emergence, sometimes even misleading people thinking that combination of various parts will deduce more. Kryazhimskiy had found "Antagonistic epitasis" explore the phenomena "the sum is less than its parts"

With the appearance of more and more unmanned facilities, intelligent weapons on the battlefield, such as unmanned aerial vehicle, unmanned ground vehicle, unmanned underwater vehicle, and the smart bomb, command and control topology which is based on the organization structure should be thoroughly transformed in order to acquire the priority on the battlefield. It is a usual combat mode that diversities form equipments are combined together to accomplish an emergent unpredictable task as was not necessary in the past. All the elements include heritage weapons, new coming ones, even some future under developing equipments taken into consideration for the potential conflict. The developing of various form military SoS becomes necessarily to the governance by the informationbased network centric war mode broadly appearing and keeping dominance in the predictable future warfare.

It is important to classify the relations between constitute systems and orchestrate elements to accomplish high-level objective from various stakeholders under such a changeable environment of SoS evolution and development. Report [5] listed seven core elements of SoS System engineering, they are

- Translating SoS Capability Objectives into High-Level SoS Requirements

- Understanding the Constituent Systems and Their Relationships

- Assessing Extent to Which SoS Performance Meets Capability Objectives

- Developing, Evolving and Maintaining an Architecture for the SoS

- Monitoring and Assessing Potential Impacts of Changes on SoS Performance

- Addressing SoS Requirements and Solution Options

- Orchestrating Upgrades to SoS

There are four items concerning with the relation structure in the above. The results of interaction and cooperation among constitute systems under certain rules bring desired capability available to SoS. The abilities and actions of individuals of SoS would be articulated by purpose architecture, the capability and performance of SoS could be compatible of solving the tasks at macro level. The emergent behaviours derived from the integration of individuals are the power force driving the SoS performance to meet the capability objectives. "Whole is more than sum of parts" is an optimal outcome of SoS evolution, but sometimes the neglect impacts will make big losses far from imagination.

\subsection{Emergence Behavior and WSoS Capability}

With regard to weapon system of systems (WSoS), a fully networked, informationbased and integrated across the Army and the joint force bring the unbelievable emergence to us. But we cannot to deduce the emergence to the new information based technology, the emergence existed long before the modern information age war, the 4th Armored Division had shown greatly agile coordination and cooperation autonomously in Second World War battlefield. The 4th Armored Division that maximized the potential of decentralized command and control was able to set overall boundaries that allowed self-organization and guided emergent behaviours. This form of cooperation and coordination, happened in battlefield, is popular applied in modern information based war. But it is hardly archived over 70 years ago with only simple walkie-talkie lacking of the support of various communication and situation awareness methods, even less of ISR (Intelligence Surveillance and Reconnaissance) equipments assisted to provide sufficient information about the 
battlefield. The case of The 4th Armored Division show that the bottom-up decentralized decision and action is the main causation for emergence, the information technology or others are only the activators which could promote the emergent behaviours.

But there are no permanent labels stuck with a certain emergent behaviour to identify its benefit or harm for the SoS during SoS evolution. Because weather benefit or not is judged only by the stage goal of SoS which is determined by the mixture of changeable strategic mission and preference of stakeholders, the high uncertainty environment and long lifecycle of SoS make the objectives varied frequently. Some emergent behaviour which were harmful yesterday perhaps support us greatly in today's situation, especially irregular combat mode has come to occupy a big partition in modern force operation, asymmetric, nonlinear, non-equilibrium, chaotic and uncertainties amount to diversity emergence in real war. The fast counter change situation take place in the battlefield makes it necessary to cope with the sprouted emergence with effective ways. And the first step is to justify its potential effects for the current and the following SoS running, then some prepared response solutions should be considered before a big shock to the SoS caused by the emergent behaviours.

We have great interests of guiding the emergence to the desired direction of stakeholders of SoS. A procedure driving to generate the advance act plan response to the impacts of emergence, promotion of beneficial emergence and reduction of harmful one, is an important part of emergence engineering. The predefined act guidance which is the results of the iterative Agent based simulation under various scenarios will provide options for the decision makers. Agent based simulation for emergent behaviours analysis is the popular ways to investigate the process of SoS evolution. Quek and Tan applied their studying the microscopic behavioural dynamics of civil violence to reveal global emergent phenomena. A formal agentbased simulation framework (FABS) was presented in, and that was demonstrated by the boids model of self-organized flocking. But most of these researches prefer to the study of collaboration, consensus and network topological, applying the classic intelligent Agent model and complex adaptive systems (CAS) theory for optimization investigation.

Huge amount agents and simple interaction rules are the usual configuration in most Agent based simulation for CAS. The independent governance, complex action of constitutes and various relationships among them make a great difference between SoS evolution simulation and normal CAS. Because the constitutes of SoS are not uniform, the legacy equipments, new facilities and something under developing are articulated into the evolutionary SoS. They show different functions, interfaces, act rules, even some unknown new characters from the future forth coming system. Simple rules among the agent running in simulation are not feasible for SoS investigation. Network based combat makes the order transfer and information share more frequently than before. The abstract logic relations between agents need to be extended to contain more meanings in order to model the real command and control in the battlefield.

Normally we cannot determine the causality of emergent behaviours from the microscopic interactions. Sometimes we even confused with which systems are the core contributors to a certain capability, because we found such capability remain its performance after one of our proposal important systems were removed from the SoS. So the connection logic modelling and constitute system modelling are hard but necessary for the investigate emergence in the SoS operation. 


\subsection{Description of WSoS CRP Problem}

2.3.1. The Assumptions of the Problem: The WSoS capability requirements are decomposable and can be aggregated. Meanwhile, the WSoS capability requirement is measurable. Interval number [5-6] is an efficient means to depict complex and uncertain information, which is widely used in multiple attribute decision-making, etc. In this paper, interval is used to represent the value of WSoS capability requirements index. The structure of the value of a capability index is shown as a pair of real numbers:

$$
q_{j}^{d i}=\left[a_{j}^{L}, a_{j}^{U}\right]^{i i}
$$

Where, $q_{j}^{d i}$ is the value of index $j$ belong to sub-capability $i$ in the level $1, l, m \hat{\mathrm{I}} N^{+}, i £ m, m$ is the number of the capabilities is the level $1, n \hat{\mathrm{I}} N^{+}, j £ n, n$ is the number of indexes of the sub-capability $i$ in the level $1 . a_{j}^{L}$ is the upper limit and $a_{j}^{U}$ is the lower limit of the interval respectively.

Thus, a sketch map of WSoS capability indexes decomposition is shown in Figure 2. A capability requirements index system can be divided into at least three levels: top capability level, sub-capability level and performance capability level. $\mathrm{S}$ is the number of levels of a WSoS capability indexes system, $s^{3} 3$ and level 1 is top capability level, level 2 to $\mathrm{s}-1$ is sub capability level and level $\mathrm{s}$ is performance capability level.

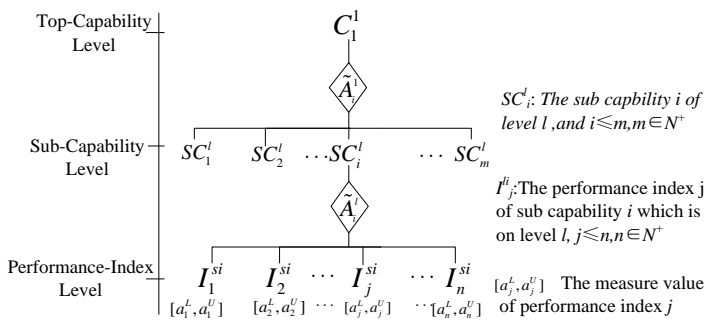

Figure 2. WSoS Capability Requirements Indexes Tree Model

2.3.2. The Inputs of the CRP Problem: The inputs of the problem are the initial interval value of the capability requirements indexes and a variety of constraints extracted from the WSoS based on the thoughts of multiple views.

The input capability requirements indexes can be expressed as a tuple Former_CIndex including the capability index identifier, capability index name and initial capability index value:

Former_CIndex $=\{$ CIndex_ID,CIndex_Name,Former_CIndex_Value $\}$

Where, CIndex_ID is the only identifier of the capability index, CIndex Name $_{-}$ represents the name of the capability index and the last Former CIndex $_{-}$Value is the capability index value.

In the application of constraint network approach, a key input is all kinds of rules, relationships, and constraints obtained from the practical problems, which will be converted to quantitative constraints with a serial of variables. Based on the thoughts of multiple views, a variety of constraints extracted from the WSoS to build a constraints network model.

2.3.3. The Output of the CRP Problem: The final output of the WSoS capability requirements planning problem is a capability requirement indexes plan to meet the given mission task requirement and the rule conditions and constraint conditions of the WSoS 
The plan is the quantitative description of the WSoS capability requirement to achieve a global goal.

\subsection{The Definition and Extraction of the WSoS Capability Index Constraints}

\section{[Definition 1] WSoS Capability Index Constraints}

WSoS capability index constraints refer to a series of needs restrictions among capability indexes. If we describe WSoS by several architecture view model (multiple views) products, the WSoS capability index constraints include all level requirements in the views products, such as the individual system stakeholders' expectations, the development levels of weapon equipment, and the technical standards of the system for collaborative tasks to achieve performance requirements.

Based on the thoughts of multiple views, the types of constraints of the WSoS capability requirements index are divided into the following five types and there are many constraints extraction methods [7-8], as shown in Table 1.

Table 1. List of Constraint s Classification

\begin{tabular}{|c|c|c|c|}
\hline Code & Class & Source & Extraction Method \\
\hline CC & Capability Constraints & Relationship between Capabilities & \\
\cline { 1 - 2 } OC & Operation Constraints & $\begin{array}{r}\text { Operation Requirements, including operation } \\
\text { activities, rules and process }\end{array}$ & $\begin{array}{r}\text { Reasoning from Data, } \\
\text { Choosing by Man-In-The- } \\
\text { Loop, Statistical Analysis } \\
\text { using Historical Data }\end{array}$ \\
SC & System Constraints & $\begin{array}{r}\text { Need of components' function and } \\
\text { Relationship between components }\end{array}$ & \\
\hline TC & Technology Constraints & Limit of Technologies and standards & \\
\hline OtC & Other Constraints & Historical Data, Experts' experience, etc & \\
\hline
\end{tabular}

\section{The Definitions of IWCN Model and the Conflicts}

\subsection{The Definition of IWCN Model}

Interval Weighted Constraint Network (IWCN) is a special CN with the domain of each variable as an interval instead of a single value and the importance of the constraints as the weights. However, the domain of IWCN variable can be real intervals [9-10] or a set of discrete values [11-12]. The mathematical expression is presented in the following:

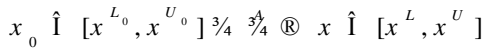

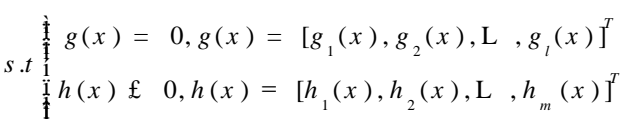

Where, $x\left(\begin{array}{lll}x \hat{\mathrm{I}} & R n\end{array}\right)$ is a constraint variable. $\mathrm{g}(\mathrm{x})$ and $\mathrm{h}(\mathrm{x})$ are both constraints function vectors, the former is used in equation but the later in inequation. $\left[x^{L}, x^{U}\right]$ is the consistent intervals of the constraint variable after the initial one $\left[x^{L_{0}}, x^{U_{0}}\right]$ is treated by constraint propagation algorithm (CPA) A, which validates the consistency of the initial interval of parameter. It is necessary to forecast the conflict and figure out the interval of variable before design optimization.

In practice, considering the characteristic of interval algorithm, the formats of constraints are required to be standardized, namely, the constraints in equation are converted into these in inequation. Assistant variable $q=\left[q_{1}, q_{2}, \mathrm{~L}, q_{m}\right]^{T}(q \mathrm{f} \quad 0)$ is added into this process, thus, the transform between inequation in expression (2) and its equation can be obtained as: 


$$
\begin{aligned}
& x_{0} \text { Î }\left[x^{L_{0}}, x^{U_{0}}\right] 3 / 43 \frac{4}{4} \text { @ } x \text { Î }\left[x^{L}, x_{U}\right] \\
& \text { s.t } \quad g(x)=0, g(x)=\left[g_{1}(x), g_{2}(x), \mathrm{L}, g_{l}(x), g_{l+1}(x), \mathrm{L}, g_{l+m}(x)\right]^{T}
\end{aligned}
$$

The computation of WSoS capability requirement index shows typical characteristics of the constraint stratification problem (CSP), which is to determine the value of each capability index $I_{s i j}$ when it fits the WSoS construction constraint conditions. Some related constraints compose the Constraint Space of indexes design environment. Take the performance indexes as an example, which can be depicted as following:

$$
\begin{aligned}
& \mathscr{q} \delta_{j}^{i} \hat{\mathrm{I}}\left[a_{j}^{L}, a_{j}^{U}\right]
\end{aligned}
$$

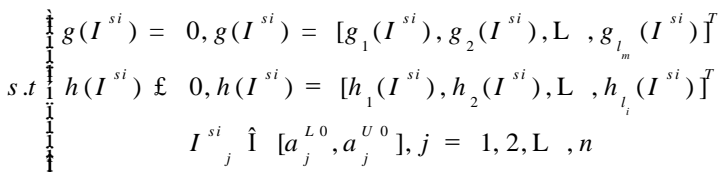

Based on the experts' experience, the importance rank of constraint $\mathrm{i}(i=1,2, \ldots, n)$ is given as $\operatorname{imp}(i)$,therefore, the importance of constraint [13-14] is defined in formula 5 .

$$
m(i)=\frac{i m p(i)}{\stackrel{\mathrm{a}}{1}_{1}^{n} \operatorname{imp} p(i)}
$$

Where, $\operatorname{imp} p(i) \hat{\mathrm{I}}[0,1], m(i) \hat{\mathrm{I}}[0,1]$, when $\operatorname{im} p(i)=0$ and $m(i)=0$,the constraint importance is so low that it can be dropped; $\operatorname{im} p(i) \hat{I}(0,1)$.the constraint importance is average and it can be dropped in proper situations. $\operatorname{im} p(i)=1$, The constraint importance is of great high level and it cannot be dropped.

\subsection{Constraint Propagation Algorithm}

There are many techniques for solving CSP. Yang C.C and Yang M.H [7-8] classify these techniques into problem reduction, solution synthesis, and searching. The technique we adopt constraint propagation which is a type of searching to solving WSoS IWCN model. Constraint propagation is a technique to maintain consistency among nodes of a constraint network. Usually, constraint propagation is embedded in a backtracking algorithm to solve a CSP. The following procedure AC3-LA is constraint propagation with consistency techniques by full-look-aheadsearch [16] which is called Maintaining Arc-consistency (MAC). It is to perform full arc consistency that will further reduce the domains and removes possible conflicts. In the AC3-LA procedure we use the AC-3 algorithm [16] as arc-consistency algorithm.

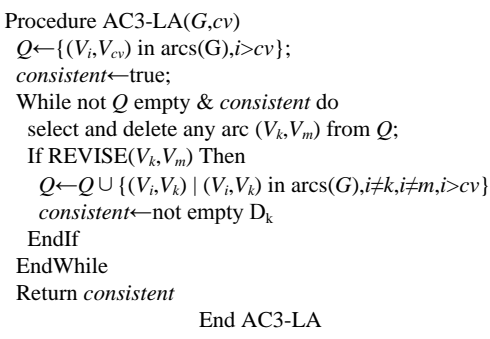

Figure 3. MAC Algorithm for Solving CSP 


\subsection{Conflicts Elimination Strategy}

The aim of conflicts elimination [13] is to search the bottlenecks of variables and adjust the interval to get optimal range of capability indexes. The novel conflicts elimination approach considers the unique characteristics of capability requirement indexes of WSoS. The main steps are as follows.

Step 1: Prioritization according to importance of
constraints.
Step 2: Check the conformance of all constraints for
each variable, determine if there are conflicts exist in
the variable interval.
Step 3: If conflicts exist, two methods can be
adopted: remain the least important conflict state or
delete the least important constraints.
Step 4: Repeat Step 2 according to determined times,
move to next step when completed.
Step 5: Obtain the result range of capability indexes
which satisfy all the constraints.

Figure 4. Conflicts Elimination Strategy Steps

\section{Case Study}

The Joint Operations System of Systems (JOSoS) based on the space force, is a new generation of armored equipment WSoS under the conditions of information warfare equipment, to meet the diversified military missions and operational tasks requirements of the new era [4]. We built the IWCN model of the JOSoS as shown in the following to solve its CRP problem.

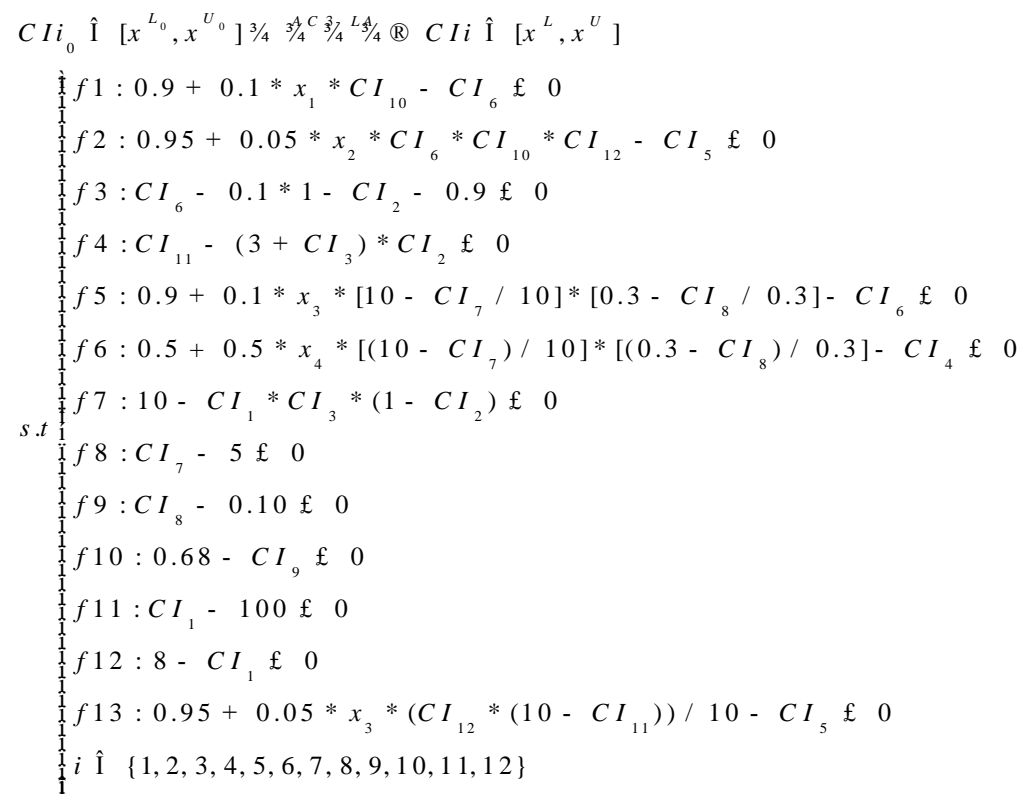

Where, CI represents the capability requirement index, the constants value are given as $x_{1}=x_{2}=x_{3}=x_{4}=x_{5}=1$, the initial and final capability requirement intervals are given in the Table and the final capability requirement intervals make the capability requirement plan. 
Table 2. The Capability Requirements Indexes of JOSoS

\begin{tabular}{|c|c|c|c|c|}
\hline $\begin{array}{c}\text { Top- } \\
\text { capability }\end{array}$ & Sub-capability & Performance index & Code & Units \\
\hline \multirow{12}{*}{ JOSoS } & \multirow{3}{*}{ Integrated Information } & Communication rate & CI1 & Mbps \\
\hline & & Communication miscounts & $\mathrm{CI} 2$ & \\
\hline & & Communication Delay & $\mathrm{CI} 3$ & $\mathrm{~s}$ \\
\hline & \multirow{2}{*}{ Firepower } & Shoot Probability & $\mathrm{CI} 4$ & \\
\hline & & Assessment Veracity & CI5 & \\
\hline & \multirow{3}{*}{ Navigation and Position } & Recognition Accurateness & CI6 & \\
\hline & & Position Accurateness & $\mathrm{CI} 7$ & $\mathrm{~m}$ \\
\hline & & Navigation Accurateness & CI8 & $\mathrm{m} / \mathrm{s}$ \\
\hline & \multirow{4}{*}{$\begin{array}{l}\text { Reconnaissance and Early- } \\
\text { warning }\end{array}$} & Anti-jamming capability & $\mathrm{CI} 9$ & \\
\hline & & Jamming antagonism validity & CI10 & \\
\hline & & Early-warning time & CI11 & $\mathrm{ms}$ \\
\hline & & Early-warning Veracity & CI12 & \\
\hline
\end{tabular}

Table 3. The Initial Interval and the Final Capability Requirement Plan

\begin{tabular}{|c|c|c|c|c|c|c|}
\hline \multirow{2}{*}{ Code } & \multicolumn{2}{|c|}{ The interval boundary } & \multicolumn{2}{c|}{ Initial intervals } & \multicolumn{2}{c|}{ Final intervals } \\
\cline { 2 - 7 } & Lower limit & Upper limit & Lower limit & Upper limit & Lower limit & Upper limit \\
\hline CI1 & 0 & 100 & 10 & 30 & 8 & 100 \\
\hline CI2 & 0 & 1 & 0 & 0.1 & 0 & 0.1 \\
\hline CI3 & 0 & 10 & 0 & 1 & 1 & 1.389 \\
\hline CI4 & 0 & 1 & 0.5 & 1 & 0.667 & 1 \\
\hline CI5 & 0 & 1 & 0.95 & 1 & 0.9878 & 1 \\
\hline CI6 & 0 & 1 & 0.9 & 1 & 0.95 & 1 \\
\hline CI7 & 0 & 10 & 0 & 5 & 0 & 5 \\
\hline CI8 & 0 & 0.3 & 0.1 & 0.15 & 0 & 0.1 \\
\hline CI9 & 0 & 1 & 0.8 & 1 & 0.68 & 1 \\
\hline CI10 & 0 & 1 & 0.8 & 1 & 0.8 & 1 \\
\hline CI11 & 0 & 10 & 0 & 3 & 0 & 3.6 \\
\hline CI12 & 0 & 1 & 0.99 & 1 & 0.995 & 1 \\
\hline
\end{tabular}

\section{Conclusions}

Capability Requirements Planning is critical for architecture design and optimization in weapon System-of-Systems (WSoS). In the traditional top-to-bottom capability requirement planning (CRP) methods cannot avoid the irrationalities and conflicts between different WSoS capability requirements indexes. Based on the analysis and description of capability requirements planning problem, Interval Weighted Constraint Network (IWCN) is introduced and a consistency detection algorithm and a conflicts elimination strategy are proposed to achieve a capabilities requirement plan satisfying the tasks requirements and the capabilities indexes constraints.

The method is validated by a case study of JOSoS. The case proved that the method is available for CRP problem of WSoS. The final plan does not only meet the needs of WSoS construction but also accord with the relationship constraints between equipment as well as that between indexes. 


\section{Acknowledgments}

Constructive suggestion and comments from anonymous reviewers are very much appreciated. The authors also would like to thank the editorial office of the journal for their diligent work.

\section{References}

[1] D. A. Fisher, "An Emergent Perspective on Inter-operation in Systems of Systems", CMU/SEI-TR-003, Pittsburgh PA:S.E.I, Carnegie Mellon University, (2006).

[2] L. Yanjing, C. Leilei, Y. Kewei, Z. Qingsong and C. Y. Wu, "Study on System of Systems capability modeling framework based on complex relationship analyzing", The 4th Annual IEEE Systems Conference, (2010).

[3] USA DoD, The DoD Dictionary of Military Terms, DoD JP 1-02, (2001).

[4] "Guide to Capability-Based Planning", Joint Systems and Analysis Group of TTCP, TR-JSA-TP3-2, (2004).

[5] H. Ishibuchi and H.Tanaka, "Multiple-objective programming in optimization of the interval objective function", European Journal of Operational Research, vol. 48, (1990).

[6] R. E. Moore, R. B. Kearfott and M. J. Cloud, "Introduction to interval analysis", Society for Industrial and Applied Mathematics in Philadelphia, (2009).

[7] C. C. Yang and M. H. Yang, "Constraint Networks: A Survey", IEEE International conference on System, Man and Cybernetics, Computational Cybernetics and Simulation, (1997).

[8] C. C. Yang, M. M. Marefat and F. W. Ciarallo, "Tolerance Analysis and Synthesis by Interval Constraint Network", Proceedings of IEEE International Conference on Robotics and Automation, (1997); Albuquerque, NM.

[9] E. Davis, "Constraint Propagation with Interval Labels", Artificial Intelligence, vol. 32, (1987).

[10] E. Hyvonen, "Constraint Reasoning Based on Interval Arithmetic: the Tolerance Propagation Approach", Artificial Intelligence, vol. 58, (1992).

[11] R. Dechter and J. Pearl, "Network-Based Heuristics for Constraint-Satisfaction Problems", Artificial Intelligence, vol. 3, (1989), pp.351-366.

[12] A. K. Mackworth and E.C. Freuder, "The Complexity of Some Polynomial Network Consistency Algorithms for Constraint Satisfaction Problem", Artificial Intelligence, vol. 25, (1985), pp. 65-74.

[13] Z. Shaotong, X. Guangleng and L. Tao, "Conflict Resolution Method Based on Interval Extendibility", Control and Decision, vol. 1, no. 19, (2004).

[14] J. Yiling, H. Jie, W. Weiming, Q. Jin and P. Yinghong, "Conflict Resolution in the Automotive Configuration Process Based on Constraints Network", Journal of Shanghai Jiao tong University, vol. 1, no. 43, (2009), pp. 119-123.

[15] X. Kunwu, Q. Fei and H. Gang, “Arc Consistency Algorithm Based on Variable Transmission”, Journal of Hubei Institute for Nationalities (Natural Science Edition), vol. 1, no. 26, (2008), pp. 61-64.

[16] B. Faltings and D. S. Haroud, "Consistency Techniques for Continuous Constraints", Constraints, vol. 2, (1996), pp. 85-118

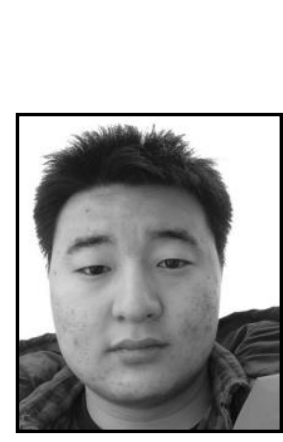

\section{Authors}

Ning Gu, he received his M.Sc. in Electronic Engineering (2011) from National University of Defense Technology. Now he is a $\mathrm{PhD}$ student at Management Department, National University of Defense Technology. His current research interests include Complex System, Logistics and Supply Chain and Decision Science.

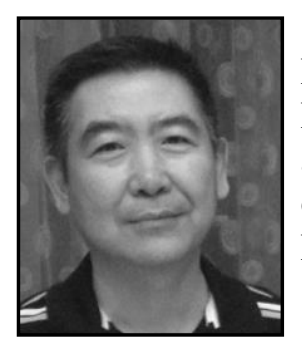

Duoyong Sun, he received his M.Sc. in Systems Engineering and Mathematics (1989) and PhD in Management Sciences (2005) from National University of Defense Technology. Now he is full professor and PhD supervisor at Management Department, National University of Defense Technology. His current research interests include Crisis Management, Complex System and Organizational Theory. 